\title{
Efeitos dos Exercícios Físicos Sobre o Edema Inflamatório Agudo em Ratos Wistar
}

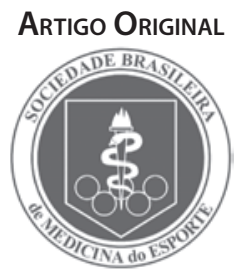

\section{Effects of Physical Exercise on the Acute Inflammatory Edema in Wistar Rats}

Ademir da Costa Lana' Célia Aparecida Paulino ${ }^{1}$ Ivair Donizeti Gonçalves'

1. Universidade Bandeirante de São Paulo (UNIBAN) - São Paulo, Brasil Departamento de Pós-Graduação

Endereço para correspondência: Rua Dr. Francisco Mesquita, 215 Apto. 44

CEP: 04304-050 - São Paulo-SP

Email:ademirlana@uol.com.br

Submetido em 22/04/2007 Versão final recebida em 30/07/2007 Aceito em 06/09/2007

\section{RESUMO}

Os exercícios físicos têm sido associados a importantes e variados benefícios à saúde, como aqueles relacionados a função imune específica e não-específica, destacando-se, nesta última, o processo inflamatório. Contudo, dependendo do tipo, intensidade, freqüência e duração, os exercícios também podem causar certos prejuízos ao organismo. De fato, estudo prévio mostrou que a hipernocicepção de origem inflamatória, em ratos, foi influenciada pelo protocolo de exercícios físicos realizados em esteira ergométrica. Assim, este trabalho teve como objetivo estudar os efeitos dos exercícios físicos de baixa e alta intensidade sobre a resposta inflamatória aguda. Para isso, foram utilizados ratos machos, adultos, da linhagem Wistar, os quais foram submetidos (grupo treinado) ou não (grupo não treinado) a exercícios em esteira ergométrica. A inflamação aguda foi induzida pela injeção de carragenina-0,5\% no coxim da pata posterior esquerda dos ratos, sendo o volume de edema inflamatório agudo mensurado por meio de pletismografia, antes e após 1, 2, 3, 4, 6, 8 e 24 horas da indução do processo inflamatório. A análise estatística dos resultados mostrou aumento significante no volume de edema inflamatório nos momentos $\mathrm{H}_{1}, \mathrm{H}_{2}$ e $\mathrm{H}_{3}(\mathrm{P}<0,01)$ e nos momentos $\mathrm{H}_{4}$ e $\mathrm{H}_{6}(\mathrm{P}<0,05)$ nos animais treinados em baixa intensidade. Entretanto, não ocorreram alterações estatisticamente significantes no volume de edema inflamatório agudo em nenhum dos momentos avaliados $(P>0,05)$ nos animais do grupo treinado em alta intensidade em relação aos não treinados. Concluiu-se, então, que os exercícios físicos de baixa intensidade, em esteira ergométrica, aumentaram o volume de edema inflamatório agudo em ratos, provavelmente ocasionado pelo aumento na síntese e secreção de prostaglandinas e/ou aumento nos níveis plasmáticos das citocinas IL-1, IL-6 e TNF-alfa entre outros fatores. Tal fato não foi observado com os exercícios de alta intensidade, mostrando assim, a influência da intensidade, freqüência e duração dos exercícios sobre este parâmetro inflamatório.

Palavras-chave: exercícios físicos, esteira ergométrica, inflamação aguda, edema, ratos.

\section{ABSTRACT}

Physical exercises have been associated with important and varied benefits to health, as those related to the specific and non-specific immune function, stressing the inflammatory process in the latter. However, depending on their type, intensity, frequency and duration, exercises can also cause certain harm to the organism. Actually, previous research has demonstrated that the hypernociception of inflammatory origin in rats was influenced by the physical exercises protocol performed on treadmill. Thus, the aim of this research was to study the effects of high and low intensity physical exercises on acute inflammatory response. Therefore, male adult Wistar rats were submitted (trained group) or not (untrained group) to physical exercises on a treadmill. Acute inflammation was induced by a $0.5 \%$ carrageenin injection into the plantar tissue of the left hind paw of each rat and the acute inflammatory edema volume was measured by plethysmography before and after 1, 2, 3, 4, 6, 8 and 24 hours of the inflammatory process induction. The statistical analysis of the results showed a significant increase in the edema volume in times $\mathrm{H}_{1}, \mathrm{H}_{2}$ and $\mathrm{H}_{3}(\mathrm{P}<0.01)$ and in times $\mathrm{H}_{4}$ and $\mathrm{H}_{6}(\mathrm{P}<0.05)$ in animals submitted to low intensity exercises. However, no statistically significant changes occurred in the acute inflammatory edema volume in any of the evaluated times ( $P>0.05$ ) in animals of the high intensity group compared with the non-trained group. Therefore, it was concluded that low-intensity physical exercises on treadmill increased the acute inflammatory edema volume in rats, probably due to the increase in the synthesis and secretion of prostaglandins and/or increase in the plasmatic levels of cytokines IL-1, IL-6 and TNF-alfa among other factors. This fact has not been observed in animals submitted to high intensity exercises, which demonstrates the influence of intensity, frequency and duration of the exercises on this inflammatory parameter.

Keywords: physical exercises, treadmill, acute inflammation, edema, rats. 


\section{INTRODUÇÃO}

A ciência tem comprovado nos últimos tempos que a prática regular de exercícios físicos tem proporcionado muitos benefícios à saúde em geral.

Durante e após os exercícios físicos ocorrem muitas modificações no sistema neuroendócrino, como: aumento dos níveis de adrenalina, noradrenalina, cortisol, hormônio liberador de corticotrofina $(\mathrm{CRH})$, hormônio adrenocorticotrófico (ACTH), entre outras substâncias endógenas, e no sistema imune, como: mudança na concentração e nas funções dos leucócitos, das células natural killer e dos linfócitos Te B, além de alterações nos níveis de imunoglobulinas, citocinas e outros fatores solúveis. Porém, a qualidade, a quantidade e o tempo necessários para essas modificações dependem da intensidade e duração destes exercícios ${ }^{(1-2)}$.

É descrito na literatura que os exercícios realizados regularmente promovem uma aceleração dos processos de reparo na inflamação ${ }^{(3)}$. Estudos evidenciaram que o exercício físico interfere em várias etapas dos processos inflamatórios, promovendo quimiotaxia e aumento da capacidade de fagocitose destas células em seres humanos e animais ${ }^{(4)}$. Resultados de Gonçalves e Luciano ${ }^{(5)}$ com ratos treinados por meio de sessões diárias de natação mostraram redução de eosinófilos circulantes e de monócitos no local inflamado, sugerindo uma atenuação da resposta inflamatória, quando comparados com ratos sedentários. Ainda, nesta direção, Lana et al. ${ }^{(6)}$ também evidenciaram redução da hipernocicepção durante a resposta inflamatória aguda induzida experimentalmente em ratos treinados em esteira ergométrica, sob um protocolo de alta intensidade, utilizando-se cerca de 70\% do consumo máximo de oxigênio $\left(\mathrm{VO}_{2}\right.$ máximo) destes animais.

Segundo Phillips et al. ${ }^{(7)}$ e Kumar et al. ${ }^{(8)}$, a resposta inflamatória ocorre no tecido conjuntivo vascularizado, no plasma, nas células circulantes, nos vasos sangüíneos e nos componentes extravasculares do tecido conjuntivo, manifestando-se pelos sinais clínicos de dor, calor, rubor e edema. Kumar et al. ${ }^{(8)}$ relataram que o edema inflamatório é a marca registrada da inflamação aguda; este fenômeno, associado ao aumento da pressão hidrostática secundária à vasodilatação, resulta em uma acentuada perda de líquidos e a um acúmulo no tecido intersticial, o que caracteriza o edema.

Ainda, conforme Garcia-Leme et al. ${ }^{(9)}$, o processo inflamatório é desenvolvido e mantido graças à liberação de mediadores químicos solúveis (aminas vasoativas, citocinas, fator de ativação plaquetária, radicais superóxidos e derivados do ácido araquidônico); destacandose, dentre estes, as citocinas ${ }^{(10)}$. Neste sentido, para Mastorakos et al. ${ }^{(11)}$, as citocinas são classificadas como antiinflamatórias ou pró-inflamatórias e, em alguns casos, com ambas ações ${ }^{(12)}$, como acontece com a interleucina 6 (IL-6). Dentre as citocinas, que parecem ser importantes mediadores na resposta inflamatória aguda, destacam-se a classe de interleucina 1 (IL-1), que consiste de três polipeptídeos estruturalmente semelhantes; os dois primeiros polipeptídeos são representados pela interleucina 1 alfa (IL-1a) e interleucina 1 beta (IL-1 $\beta)$ e a terceiro é representado pelo antagonista de receptor de interleucina 1 (IL-1 ra), além da IL-6 e o fator de necrose tumoral alfa (TNF-a) ${ }^{(13)}$.

Também é relatado que os exercícios físicos agudos podem levar a respostas do organismo, entre elas, as reações de estresse envolvendo a ativação do eixo-hipotálamo-hipófise-adrenal, o que induz liberação de $\mathrm{CRH}, \mathrm{ACTH}$ e estimulação das glândulas adrenais e a conseqüente síntese e secreção de hormônios glicocorticóides, os quais promovem adaptações metabólicas no organismo ${ }^{(14)}$, tais como a inibição da resposta inflamatória(15). Em adição, Watanabe et al. ${ }^{(16)}$ relataram que os níveis plasmáticos de ACTH podem estar aumentados em condições infecciosas e inflamatórias ou em condições de estresse, como, por exemplo, durante os exercícios físicos, devido a uma resposta induzida pela liberação de IL-1, que ocorre nestas condições. Sobre este aspecto, Sothmann et al. ${ }^{(14)}$ relataram que durante exercícios leves a secreção de cortisol, em seres humanos, é pouco alterada, mas durante exercícios exaustivos ocorre um significante aumento deste hormônio.
Ainda, vários autores sugerem uma ação adicional do $\mathrm{CRH}$, devido a presença de possíveis sítios de ligação deste hormônio detectado em tecidos periféricos, como nervos ${ }^{(17)}$, endotélio ${ }^{(18)}$ e em células do sistema imune, incluindo leucócitos circulantes ${ }^{(19)}$. A administração local e sistêmica de $\mathrm{CRH}$ promoveu redução do extravasamento plasmático em modelos animais de inflamação induzida por injúria térmica ${ }^{(20)}$. Deste modo, o CRH pode induzir efeitos antiinflamatórios, tanto diretamente, pela ativação dos seus receptores periféricos, como indiretamente, pela ativação do eixo hipófise-adrenal(21).

Diante destas informações, o objetivo deste trabalho foi o de utilizar modelos experimentais com animais de laboratório para estudar os efeitos dos exercícios físicos programados e controlados, de baixa e alta intensidade, em esteira ergométrica, sobre a evolução do edema inflamatório agudo, uma vez que não existem estudos sobre este tema descritos na literatura até o momento.

\section{MÉTODOS}

\section{Animais}

Para o desenvolvimento deste trabalho foram utilizados ratos da linhagem Wistar, machos, com aproximadamente 60 dias de idade e peso corporal variando entre 180 a 250 gramas (no início dos experimentos), adquiridos do Biotério Central da Universidade Federal de São Paulo (UNIFESP, São Paulo). Estes animais foram utilizados em conformidade com as normas e procedimentos éticos vigentes para a experimentação animal, e este estudo foi previamente analisado e aprovado pelo Comitê de Ética da Universidade Bandeirante de São Paulo - UNIBAN, São Paulo (protocolo nº 024/2004).

Os animais foram alojados em caixas plásticas de polipropileno medindo $41 \times 34 \times 16 \mathrm{~cm}$, contendo cinco ratos por caixa, e mantidos em sala de manutenção de ratos, com temperatura e umidade ambiente controladas, sob iluminação artificial com fotoperíodo de 12 horas de claro e 12 horas de escuro, iniciando-se a fase clara às 8:00 horas. Os ratos foram alimentados com ração padrão e água ad libitum, identificados, pesados e divididos aleatoriamente em grupos de animais treinados e grupos de animais não treinados. Estes animais foram mantidos nestas condições por um período de adaptação de, no mínimo, sete dias antes do início dos experimentos.

\section{Treinamento físico de baixa e alta intensidade dos ratos}

Para aplicação dos exercícios físicos de baixa e alta intensidade, os animais dos 2 grupos treinados ( $T R, n=12$ em cada grupo) foram submetidos a protocolos experimentais realizados em esteira ergométrica programável e adaptada para ratos (10400-Inbramed ${ }^{\circledR}$ ), com 8 raias individuais de aço inox, providas de orifícios para ventilação e cobertas individualmente por tampas móveis de acrílico transparente, com orifícios para ventilação. Os ratos dos 2 grupos não treinados (NTR, n=12 em cada grupo) foram apenas manipulados e colocados na esteira (sem movimento), com a finalidade de exposição ao local dos exercícios (esteira ergométrica), que pode representar uma fonte de estresse para estes animais.

O protocolo de exercícios físicos em baixa intensidade constituiu-se de um treinamento com duração de 12 semanas, 5 vezes por semana. Nas duas primeiras semanas, a duração das sessões foi aumentada gradativamente até 60 minutos por dia e mantida até o final do treinamento; a velocidade foi de $5 \mathrm{~m} / \mathrm{min}$ nas duas semanas iniciais e também foi aumentada gradativamente até atingir a velocidade de $15 \mathrm{~m} / \mathrm{min}$. A inclinação da esteira ergométrica foi mantida a $1 \%$ durante todo o período do treinamento.

O protocolo de exercícios físicos em alta intensidade também foi constituído de um treinamento progressivo com duração de 11 semanas, 5 vezes por semana. Na primeira semana os animais foram colocados na esteira ergométrica e treinados durante 15 minutos, 5 $\mathrm{m} / \mathrm{min}$, com $0 \%$ de inclinação. A partir desta primeira semana até o final da sexta semana, a duração, velocidade e inclinação da esteira foram 
aumentadas gradativamente até 75 minutos de duração, velocidade de $25 \mathrm{~m} / \mathrm{min}$ e $15 \%$ de inclinação, esta última mantida por mais um período de 5 semanas.

Apesar de não serem diretamente mensuradas, as intensidades dos exercícios físicos de baixa e alta intensidade foram baseadas em estudos prévios, que estimaram um $\mathrm{VO}_{2}$ máximo de aproximadamente $50 \%$ para os exercícios de baixa intensidade ${ }^{(22-23)}$ e de cerca de $70 \%$ para os de alta intensidade(22-24).

\section{Indução da resposta inflamatória aguda}

A resposta inflamatória aguda foi induzida pela injeção de uma suspensão do irritante carragenina tipo Lambda (Sigma ${ }^{\circledR}$ ) na concentração de 0,5\%, diluída em solução de Ringer (Aster ${ }^{\circledR}$ ), que provoca uma reação inflamatória rápida e facilmente mensurável. O agente irritante foi injetado no tecido celular subcutâneo do coxim da pata posterior esquerda de cada rato, no volume de 0,1 ml por animal. A solução restante não era utilizada em outro experimento.

\section{Medida do volume de edema inflamatório agudo}

O volume do edema inflamatório resultante no coxim plantar de cada rato foi medido pelo método da pletismografia, utilizando-se um pletismógrafo digital (Plethysmometer - Ugo Basile ${ }^{\circledR}$ ); para isso as mensurações aconteceram antes e 1, 2, 3, 4, 6, 8 e 24 horas após a injeção da carragenina ou (não)(25). Os resultados finais obtidos, ou seja, a diferença entre os volumes medidos antes e depois das injeções foi expressa diretamente em mililitros $(\mathrm{ml})$.

\section{Análise Estatística}

Os resultados obtidos foram submetidos ao teste de Bartlett para definição do tipo de teste (paramétrico ou não paramétrico). Para análise das possíveis diferenças entre os vários grupos estudados foi utilizado o teste de Análise de Variância (ANOVA), seguida do teste de Tukey-Kramer de comparações múltiplas (dados paramétricos). Utilizouse também o teste " $\mathrm{t}$ " de Student (dados paramétricos) e o teste de Mann-Whitney (dados não paramétricos), para detecção de possíveis diferenças entre os 2 grupos distintos (TR e NTR). Os resultados foram expressos como média \pm desvio-padrão e o nível de significância estatística adotado foi o de 5\% ( $<<0,05)$.

\section{RESULTADOS}

A Tabela 1 apresenta e a Figura 1 ilustra os efeitos dos exercícios físicos de baixa intensidade sobre o volume de edema inflamatório agudo; observa-se que a injeção de carragenina provocou um aumento significante $(\mathrm{P}<0,05)$ no volume de edema da pata, que variou com o tempo; o volume máximo de edema ocorreu no momento $\mathrm{H}_{4}$, após o início do experimento para ambos os grupos. A análise estatística revelou um aumento do volume de edema inflamatório nos momentos $\mathrm{H}_{1}, \mathrm{H}_{2}$ e $\mathrm{H}_{3}(\mathrm{P}<0,01)$ e nos momentos $\mathrm{H}_{4}$ e $\mathrm{H}_{6}(\mathrm{P}<0,05)$ nos animais do grupo TR em relação àqueles do grupo NTR.

Da mesma forma, a Tabela $\mathbf{2}$ apresenta e a Figura $\mathbf{2}$ ilustra os efeitos dos exercícios físicos de alta intensidade sobre o volume de edema; também houve um aumento significante $(P<0,05)$ no volume de edema da pata, que variou com o tempo e foi máximo entre os momentos $\mathrm{H}_{3}$ e $\mathrm{H}_{4}$. Todavia, diferentemente do treinamento físico em baixa intensidade, a análise estatística não revelou diferenças no volume de edema inflamatório em nenhum dos momentos analisados $(P>0,05)$ nos animais do grupo TR em relação àqueles do grupo NTR.

\section{DISCUSSÃO}

Nos últimos anos, a prática de exercícios físicos vem ganhando cada vez mais destaque por favorecer a saúde e proporcionar melhor

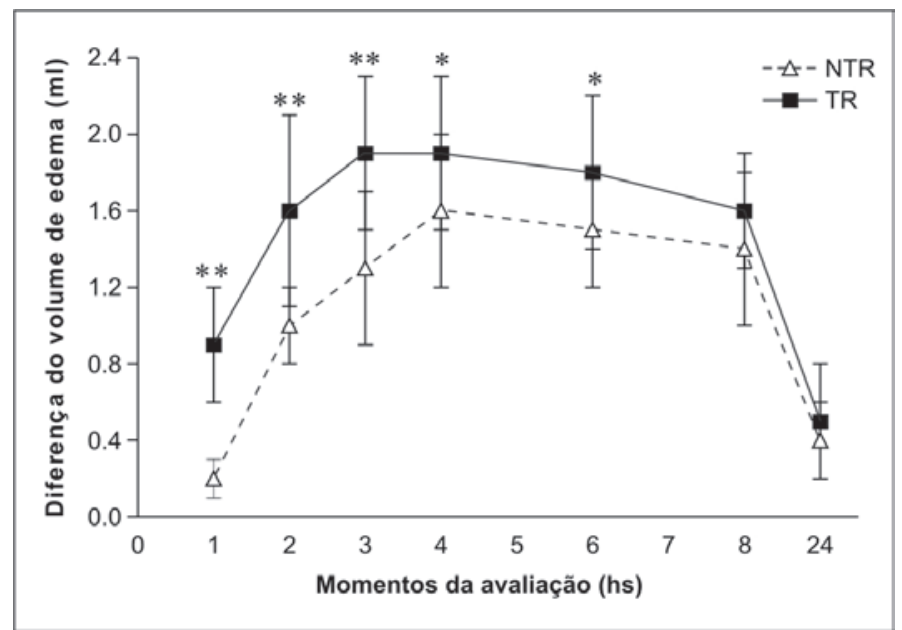

Figura 1. Diferença do volume de edema inflamatório da pata de ratos submetidos ou não a exercícios físicos de baixa intensidade em esteira ergométrica (em relação ao momento $\mathrm{H}_{0}$ ).

${ }^{*} \mathrm{P}<0,05$ (em relação ao grupo NTR).

${ }^{* *} \mathrm{P}<0,01$ (em relação ao grupo NTR).

Resultados expressos como média e desvio-padrão, n=12 por grupo.

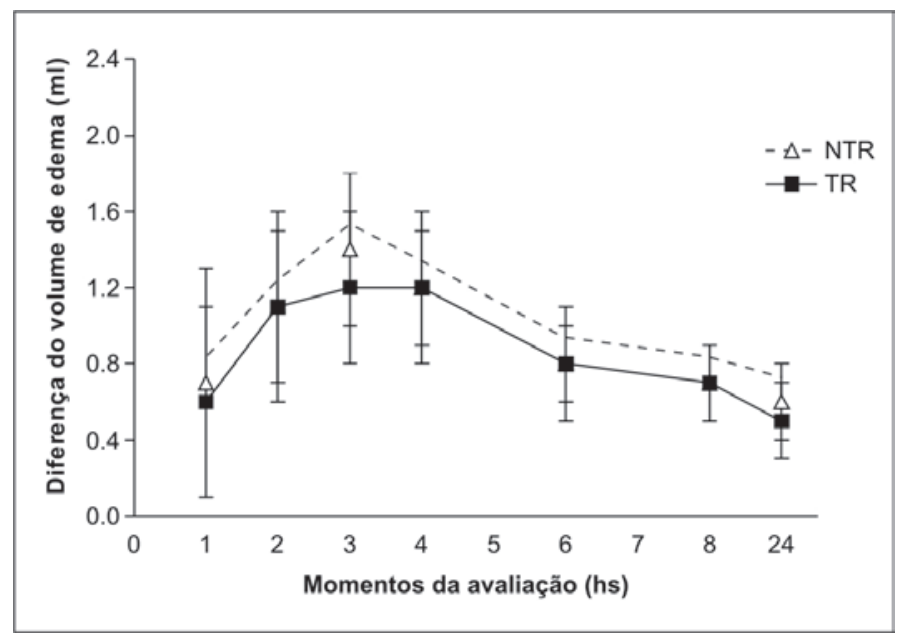

Figura 2. Diferença do volume de edema inflamatório da pata de ratos submetidos ou não a exercícios físicos de alta intensidade em esteira ergométrica (em relação ao momento $\mathrm{H}_{0}$ ).

Obs:: Não houve diferenças estatisticamente significantes entre os grupos TR e NTR. Resultados expressos como média e desvio-padrão, $n=12$ por grupo.

Tabela 1. Diferença do volume de edema inflamatório da pata de ratos submetidos ou não a exercícios físicos de baixa intensidade em esteira ergométrica (em relação ao momento $\mathrm{H}_{0}$ ).

\begin{tabular}{|c|c|c|c|c|c|c|c|}
\hline \multirow{2}{*}{ Grupos de animais $(n=12)^{a}$} & \multicolumn{7}{|c|}{ Média e desvio-padrão da diferença do volume de edema da pata (em mililitros) } \\
\hline & $\mathrm{H}_{1}$ & $\mathrm{H}_{2}$ & $\mathrm{H}_{3}$ & $\mathrm{H}_{4}$ & $\mathrm{H}_{6}$ & $\mathrm{H}_{8}$ & $\mathrm{H}_{24}$ \\
\hline TR & $0,9 \pm 0,3^{* *}$ & $1,6 \pm 0,5^{A * *}$ & $1,9 \pm 0,4^{\mathrm{A**}}$ & $1,9 \pm 0,4^{A *}$ & $1,8 \pm 0,4^{\mathrm{A} *}$ & $1,6 \pm 0,3^{A C D}$ & $0,5 \pm 0,3^{E}$ \\
\hline NTR & $0,2 \pm 0,1$ & $1,0 \pm 0,2^{A}$ & $1,3 \pm 0,4^{\mathrm{B}}$ & $1,6 \pm 0,4^{\mathrm{B}}$ & $1,5 \pm 0,3^{\mathrm{B}}$ & $1,4 \pm 0,4^{\mathrm{B}}$ & $0,4 \pm 0,2^{F}$ \\
\hline
\end{tabular}

a número de animais por grupo.

*P<0,05 em relação ao grupo NTR (teste de Mann-Whitney).

**P $<0,01$ em relação ao grupo NTR (teste "t" Student não pareado).

Ap $<0,05$ em relação ao momento $H_{1}$ (ANOVA seguida de Tukey-Kramer).

${ }^{\mathrm{B}} \mathrm{P}<0,05$ em relação ao momento $\mathrm{H}_{1}$ até $\mathrm{H}_{2}$ (ANOVA seguida de Tukey-Kramer).
${ }^{C} \mathrm{P}<0,05$ em relação ao momento $\mathrm{H}_{3}$ (ANOVA seguida de Tukey-Kramer).

$\mathrm{DP}<0,05$ em relação ao momento $\mathrm{H}_{4}$ (ANOVA seguida de Tukey-Kramer).

EP $<0,05$ em relação ao momento $H_{1}$ até $H_{8}$ (ANOVA seguida de Tukey-Kramer).

${ }^{\mathrm{F}}<0,05$ em relação ao momento $\mathrm{H}_{2}$ até $\mathrm{H}_{8}$ (ANOVA seguida de Tukey-Kramer). 


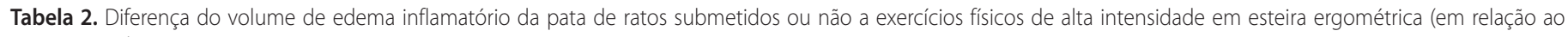
momento $\mathrm{H}_{0}$ ).

\begin{tabular}{c|c|c|c|c|c|c|c}
\hline \multirow{2}{*}{ Grupos de animais $(\mathbf{n}=12)^{\mathrm{a}}$} & \multicolumn{6}{|c}{ Média e desvio-padrão da diferença do volume de edema da pata (em mililitros) } \\
\cline { 2 - 8 } & $\mathbf{H}_{1}$ & $\mathbf{H}_{2}$ & $\mathbf{H}_{3}$ & $\mathbf{H}_{4}$ & $\mathbf{H}_{6}$ & $\mathbf{H}_{\mathbf{8}}$ & $\mathbf{H}_{24}$ \\
\hline TR & $0,6 \pm 0,5$ & $1,1 \pm 0,4^{\mathrm{A}}$ & $1,2 \pm 0,4^{\mathrm{A}}$ & $1,2 \pm 0,4^{\mathrm{A}}$ & $0,8 \pm 0,3^{\mathrm{B}}$ & $0,7 \pm 0,2^{\mathrm{C}}$ & $0,5 \pm 0,2^{\mathrm{D}}$ \\
\hline NTR & $0,7 \pm 0,6$ & $1,1 \pm 0,5^{\mathrm{A}}$ & $1,4 \pm 0,4^{\mathrm{A}}$ & $1,2 \pm 0,3^{\mathrm{A}}$ & $0,8 \pm 0,2^{\mathrm{B}}$ & $0,7 \pm 0,2^{\mathrm{C}}$ & $0,6 \pm 0,2^{\mathrm{C}}$ \\
\hline
\end{tabular}

a número de animais por grupo.

AP $<0,05$ em relação ao momento $H_{1}$ (ANOVA seguida de Tukey-Kramer).

${ }^{B} \mathrm{P}<0,05$ em relação ao momento $\mathrm{H}_{3}$ até $\mathrm{H}_{4}$ (ANOVA seguida de Tukey-Kramer).

${ }^{C} \mathrm{P}<0,05$ em relação ao momento $\mathrm{H}_{2}$ até $\mathrm{H}_{6}$ (ANOVA seguida de Tukey-Kramer).

$\mathrm{DP}<0,05$ em relação ao momento $\mathrm{H}_{2}$ até $\mathrm{H}_{4}$ (ANOVA seguida de Tukey-Kramer).

Obs.: Não houve diferenças estatisticamente significantes entre os grupos TR e NTR (teste "t" Student não pareado).

qualidade de vida à população. Os exercícios praticados regularmente levam a adaptações fisiológicas e morfológicas importantes para a manutenção da homeostasia ${ }^{(26)}$, e essas adaptações são importantes para o controle de muitas doenças, em especial as de natureza cardiovascular e endócrino-metabólica ${ }^{(27)}$.

Apesar destes efeitos benéficos, durante ou após os exercícios físicos ocorre um aumento do consumo de oxigênio e ativação de vias metabólicas específicas, o que resulta na formação de radicais livres de oxigênio ${ }^{(28-29)}$. Conforme Southorn e Powis ${ }^{(30)}$, essas moléculas de radicais livres estão aumentadas principalmente nos exercícios de alta intensidade e foram relacionadas ao envelhecimento e a um grande número de doenças, tais como: enfisema pulmonar, doenças inflamatórias e câncer. Também, os exercícios físicos são uma conhecida forma de estresse, capaz de disparar adaptações em resposta a uma maior produção de radicais livres ${ }^{(31)}$, gerando uma série de alterações bioquímicas, psicológicas e comportamentais(32)

Sobre este aspecto, vários estudos realizados em seres humanos e ratos, reforçam o conceito de que os exercícios físicos, em suas diversas modalidades, são capazes de alterar várias funções do organismo, como aquelas relacionadas ao sistema neuroendócrino. Neste sentido, Lana et al.(6) relataram diminuição do limiar de hipernocicepção, de origem inflamatória, em ratos treinados em alta intensidade em esteira ergometria, provavelmente por alterações no sistema neuroendócrino, entre outros fatores. Todavia, os estudos publicados até o momento mostram grandes discrepâncias de resultados, por várias razões, como a utilização de diferentes protocolos de treinamento físico (forçado ou voluntário), a variação no tipo, duração, freqüência e intensidade destes exercícios, bem como a multiplicidade de testes utilizados para avaliação da influência dos exercícios físicos nos parâmetros investigados ${ }^{(33)}$.

De fato, alterações neuroendócrinas podem ocorrer em seres humanos e animais após exercícios físicos e, para isso, são normalmente utilizadas sessões de corrida, ciclismo e natação em humanos, e a natação forçada em ratos ${ }^{(34)}$. Assim, é importante a utilização de outros tipos de exercícios com estes animais, como por exemplo, aqueles realizados em esteira ergométrica. Também, a comparação dos efeitos dos exercícios em diferentes intensidades, como os utilizados neste trabalho, é relevante na tentativa de contribuir com dados ainda escassos na literatura em relação ao edema inflamatório. Ainda, a busca por tratamentos mais seguros, em substituição aos fármacos antiinflamatórios, confere à prática de exercícios físicos um lugar de destaque como importante possibilidade terapêutica.

Considerando que os exercícios físicos representam uma forma de estímulo estressante ${ }^{(35-36)}$ capaz de promover mudanças na homeostasia com a reorganização das respostas de diversos sistemas, entre eles o sistema neuroendócrino ${ }^{(37)}$, este trabalho avaliou a influência dos exercícios físicos sobre a modulação da resposta inflamatória aguda, representada pelo edema inflamatório em ratos submetidos a exercícios físicos de baixa e alta intensidade, em esteira ergométrica.

A inflamação é uma resposta do organismo às agressões e é caracterizada, em sua forma aguda, pelos sinais clássicos de dor, rubor, edema e perda da função ${ }^{(38)}$. Segundo Garcia-Leme ${ }^{(39)}$, o edema inflamatório agudo é um sinal indicativo de injúria das células endoteliais e é produzido pela liberação seqüencial de mediadores químicos, como: histamina, serotonina (5-HT), citocinas e prostaglandinas ( $P G^{\prime}$ s), estas últimas com um papel crucial neste processo, embora os hormônios glicocorticóides endógenos também desempenhem importante função no controle da inflamação. Portanto, de acordo com Martinez e Bolaños ${ }^{(40)}$, no desenvolvimento do edema inflamatório agudo induzido pela carragenina na pata de ratos ocorre, inicialmente (ao redor da primeira hora), uma liberação de histamina e de $5 \mathrm{H}-\mathrm{T}$, seguida pela de bradicinina (ao redor da segunda hora) e, posteriormente, pela de PG's (ao redor de 3 a 6 horas), que ajudam a manter o pico deste edema.

O volume do edema gerado aumenta gradativamente em função do tempo, atingindo um pico máximo por volta de 2 a 4 horas após a injeção da carragenina ${ }^{(9-41)}$. 0 edema medido no presente trabalho coincidiu com dados da literatura ${ }^{(9-25)}$ ao evidenciar que a injeção de $0,1 \mathrm{ml}$ de suspensão de carragenina a 0,5\% no coxim plantar dos ratos, provocou um edema cujo volume aumentou gradativamente em função do tempo, com volume máximo por volta de 2 a 4 horas após a injeção do irritante.

Desta forma, os resultados encontrados indicam que os exercícios físicos de baixa intensidade, em esteira ergométrica, foram capazes de causar aumento estatisticamente significante no volume de edema inflamatório agudo da pata dos ratos treinados nos momentos $\mathrm{H}_{1}$ até $\mathrm{H}_{6}$, com volume máximo no momento $\mathrm{H}_{4}$, em relação àqueles não treinados. Como os agentes antiinflamatórios são capazes de diminuir o edema induzido pela carragenina e outros sinais inflamatórios através da inibição da síntese de PG's ${ }^{(9-42-43)}$, é possível sugerir que estes resultados são conseqüentes ao aumento na síntese e liberação destas substâncias.

É sabido que a produção de PG's é estimulada, entre outros agentes, por IL-1 e TNF-a ${ }^{(44)}$. Adicionalmente, sabe-se que em resposta aos exercícios físicos ocorre um aumento significativo nos níveis de várias citocinas ${ }^{(12)}$, provavelmente associadas às contrações ou lesões musculares, e de TNF-a, que precede e estimula a liberação de IL-1, a qual atua de maneira sinérgica estimulando a produção de IL-6; todas estas reações ocorrem de maneira seqüencial ${ }^{(10-45-46)}$. Além disto, Bury et al. ${ }^{(47)}$ relataram que o aumento nos níveis de IL-1 estão diretamente relacionados com a intensidade do exercício físico; neste sentido, constataram que quando indivíduos treinavam utilizando 45, 60 e 75\% do seu $\mathrm{VO}_{2}$ máximo, durante 4, 3 e 2 horas, respectivamente, ocorria um aumento progressivo correspondente nos níveis de IL-1 de 60, 97 e 142\% ao final dos exercícios físicos. Assim, é possível afirmar que o aumento no nível plasmático destas citocinas, ocorridas durante a realização dos exercícios de baixa intensidade, foi responsável pelo aumento na síntese e secreção de PG's, que promoveram elevação no volume do edema da pata destes animais.

Em contrapartida, não houve alteração do volume de edema da pata dos ratos submetidos aos exercícios de alta intensidade, em nenhum dos momentos avaliados. Como citado anteriormente, o aumento nos níveis de IL-1 está diretamente relacionado com a intensidade dos exercícios ${ }^{(47)}$. Outro fator a ser considerado é a presença de altos níveis de IL-1 e IL-6, que induzem a síntese e liberação de IL-1ra, um inibidor específico das atividades das IL's-1 (IL-1 a e IL-1 $\beta$ ), atuando de forma a bloquear sua 
ligação ao receptor localizado na superfície celular, protegendo, desta forma, o organismo contra seus efeitos exacerbados ${ }^{(10-46)}$.

Nesta mesma direção, Ostrowiski et al. ${ }^{(45)}$ observaram que após uma corrida de maratona houve um aumento de até 2 vezes nos níveis plasmáticos de IL-1 $\beta$ e TNF-a e até 100 vezes nos níveis de IL-6, que estimula um aumento acentuado nos níveis de IL-1ra. Em concordância, de acordo com Ostrowski et al. ${ }^{(48)}$, o aumento nos níveis de IL-1 $\beta$ e TNF-a é acompanhado por uma elevação nos níveis de IL-6, cuja síntese é balanceada por citocinas de caráter inibitório, como a IL-1 ra, e por citocinas de caráter antiinflamatório, como a interleucina-10 (IL-10). Deste modo, estes resultados podem indicar que altos níveis plasmáticos de IL-1 estimulados pelos exercícios de alta intensidade sofreram ação inibitória da IL-1ra. Também, a IL-6 é capaz de atuar como antiinflamatória ou pró-inflamatória, ou ambas ações, mas há evidências que indicam que esta interleucina possui primariamente efeitos antiinflamatórios ${ }^{(12)}$.

Em adição, estudos em ratos sugeriram que o aumento nos níveis de adrenalina que ocorre em situações de estresse é o responsável pelo aumento nos níveis de IL-6 $6^{(49)}$. Portanto, nos exercícios físicos de alta intensidade, o aumento desta interleucina poderia ter desencadeado uma ação antiinflamatória, não o suficiente para atenuar o edema inflamatório, mas de maneira a contrabalançar os efeitos pró-inflamatórios exacerbados, sobretudo das IL's-1.

Enfatizando os resultados aqui obtidos, Turnbull e Rivier ${ }^{(50)}$ relataram que as citocinas, em particular IL-1, IL-6 e TNF-a são potentes ativadores do eixo hipotálamo-hipófise-adrenal, quando o organismo é exposto a altos e prolongados níveis destas citocinas em situações que envolvam infecções virais e bacterianas, lesão tecidual e inflamação, além de estresse físico e psicológico. Isso resulta na liberação dos hormônios CRH, ACTH e glicocorticóides, substâncias que participam do controle da inflamação promovendo alterações nas reações vasculares aos mediadores químicos e, desta forma, redução na permeabilidade vascular e no volume de edema inflamatório ${ }^{(51)}$. Desta forma, talvez o treinamento físico de alta intensidade aqui utilizado seja um estímulo suficientemente estressor aos animais e, assim, capaz de desencadear respostas mais evidentes, principalmente aquelas envolvendo a ativação do eixo hipotálamo-hipófise-adrenal, e gerando reações que resultem em aumento nas concentrações séricas de glicocorticóides endógenos, quando comparadas ao treinamento físico de baixa intensidade.

De acordo com Terman et al. ${ }^{(52)}$ e Gamaro et al.(32), exposições repetidas ao mesmo estímulo aversivo podem levar a um processo de adaptação a este estímulo. Neste trabalho, os ratos treinados com exercícios de baixa intensidade podem ter desenvolvido uma adaptação a tais exercícios e, conseqüentemente, uma menor ativação do eixo neuroendócrino. Todos esses dados reforçam a hipótese de que os exercícios físicos de alta intensidade apresentam-se como uma forma de agente estressor potente capaz de ativar tais mecanismos, contrariamente aos exercícios de baixa intensidade.

Apesar dos modelos experimentais aqui utilizados serem descritos na literatura e, portanto, serem padronizados, este trabalho apresenta como limitações o fato de não terem sido quantificados os níveis de hormônios e citocinas, que poderiam também estar modificados em função dos exercícios aplicados, bem como os resultados aqui obtidos podem ser limitados para a extrapolação aos seres humanos, em razão do uso de modelos animais.

Finalmente, analisando-se os resultados aqui obtidos pode-se concluir que o tipo, a intensidade, a freqüência e a duração dos exercícios físicos, além de outros fatores, podem alterar o volume de edema inflamatório agudo, principalmente devido a alterações no sistema neuroendócrino, porém de modo dependente do protocolo de exercícios físicos utilizados.

Todos os autores declararam não haver qualquer potencial conflito de interesses referente a este artigo.

\section{REFERÊNCIAS BIBLIOGRÁFICAS}

1. Nieman DC. Exercise immunology: practical applications. Int J Sports Med 1997; 18: S91-100.

2. Marcus BH, King TK, Clark MM, Pinto BM, Bock BC. Theories and techniques for promoting activity behaviours. Sports Med 1996; 22: 321-31

3. Nieman DC. Influence of carbohydrate on the immune response to intensive prolonged exercise. Exerc Immunol 1998: 4:64-76.

4. Fehr $\mathrm{HG}$, Lotzerich $\mathrm{H}$, Michna $\mathrm{H}$. Human macrophage function and physical exercise: phagocytic and histochemical studies. Eur J Appl Physiol 1989; 58: 613-17.

5. Gonçalves AL, Luciano E. Respostas inflamatórias em ratos Wistar submetidos à atividade física. Rev Bras Ativ Fís Saúde 1999; 4: 39-46

6. Lana AC, Paulino CA, Donizeti, IG. Influência dos exercícios físicos de baixa e alta intensidade sobre o limiar de hipernocicepção e outros parâmetros em ratos. Rev Bras Med Esporte 2006; 12: 248-254.

7. Phillips J, Murry P, Crocker J. The biology of disease. England: Blackwell Science, 1995; 22-9.

8. Kumar V, Abbas AK, Fausto N. Robbins e Cotran: bases patológicas das doenças. Rio de Janeiro: Elsevier, 2005: 49-89.

9. Garcia-Leme J, Hamamura L, Leite MP, Rocha e Silva M. Pharmacological analysis of the acute inflammatory process induced in the rat's paw by local injection of carrageenan and by heating. Br J Pharmac 1973; 48: 88-96.

10. Dinarello CA. Wolf SM. The role of interleukin-1 in disease. N Engl J Med 1993; 328: 106-13.

11. Mastorakos $\mathrm{G}$, Chrousos GP, Weber JS. Recombinant interleukin-6 activates the hypothalamic-pituitary-adrenal Mastorakos G, Chrousos GP, Weber JS. Recombinant interleuk

2. Pedersen BK. Exercise and cytokines. Immunol Cell Biol 2000; 78: 532-5.

13. Dinarello CA. Interleukin-1 and interleukin-1 antagonist. Blood 1991; 77: 1627-52.

14. Sothmann MS, Hart BA, Horn TS. Exercise training and cross-stressor adaptation hypothesis. Exerc Sports Sci Rev 1996; 24: 267-87.

15. Prada FJ, Carneiro EM, Azevedo JRM, Luciano E. Respostas endócrino-metabólicas em ratos diabéticos. Rev Bras Ativ Fís Saúde 1997; 2: 22-9

16. Watanabe T, Morimoto A, Sakata Y, Murakami N. ACTH response induced by interleukin-1 is mediated by CRF secretion stimulated by hypothalamic PGE. Experientia1990; 46: 481-4.

17. Udelsman R, Harwood J, Millan M, Chrousos G, Goldstein D, Zimlichman R et al. Functional corticotropin releasing receptors in the primate peripheral nervous system. Nature 1986; 319: 147-52

18. Dashwood M, Andres H, Wei E. 125 -Corticotropin-releasing factor to rabbit aorta is reduced by removal of endothelium. Eur J Pharmacol 1987; 135: 111-4.

19. Singh V, Fudenberg H. ${ }^{125}$-Corticotropin-releasing factor to blood immunocytes and its reduction in Alzheimer's disease. Immunol Lett 1988; 18: 5-8.

20. Kiang J, Wei E. Corticotropin-releasing factor inhibits thermal injury. J Pharmacol Exp Ther 1987; 243: 517-9.

21. Hargreaves KM, Dubner R, Costello AH. Corticotropin releasing factor (CRF) has a peripheral site of action for antinociception. Eur J Pharmacol 1989; 170: 275-9.

22. Brooks GA, White TP. Determination of metabolic and heart rate responses of rats to treadmill exercise. J Appl Physiol: Respirat Environ Exerc Physiol 1978; 45: 1009-15.

23. Silva GJJ. Efeito do treinamento físico de baixa intensidade sobre a sensibilidade dos aferentes pressorreceptores, barorreflexo e reflexo cardiopulmonar em ratos espontaneamente hipertensos (Dissertação de Mestrado). São Paulo: Universidade de São Paulo, 1999.

24. Ramires PR. Efeitos do treinamento físico e da dieta suplementada com glutationa na resposta à isquemiareperfusão cardíaca in vivo no rato (Tese de doutorado). São Paulo: Universidade de São Paulo, 1999.

25. Costello AH, Hargreaves KM. Suppression of carrageenan-induced hyperalgesia, hyperthermia and edema by bradykinin antagonist. Eur J Pharmacol 1989; 71:259-63

26. Cartee GD, Farrar RP. Exercise training induces glycogen sparing during exercise by old rat. J Appl Physiol $1988 ; 64: 259-65$.

27. Luciano $\mathrm{E}$, Lima FB, Luciano EA. Effect of physical training on evolution of carbohydrate metabolism in diabetic rats. Med Sci Sports Exerc 1994; 26: 70-5.

28. Halliwell B, Gutteridge JMC. Free radicals in biology and medicine. New York: Oxford, 1999.

29. Rowlands DS, Downey B. Pysiology of triathlon. In: Garret WE Jr, Kirkendall DT, editors. Exercise and sports science. Philadelphia: Lippincott Willians \& Wilkins, 2000; 921-2.

30. Southron PA, Powis G. Free radicals in medicine. Involvement in human disease. Mayo Clin Proc 1988; 63: 390-408.

31. Schneider CD, Oliveira AR. Radicais livres de oxigênio e exercício: mecanismos de formação e adaptação ao treinamento físico. Rev Bras Med Esporte 2004; 10: 308-13.

32. Gamaro GD, Xavier MH, Denardin JD, Pilger J, Ely DR, Ferreira MBC, et al. The effects of acute and repeated restraint stress on the nociceptive response in rats. Physiol Behav 1998; 63:693-7.

33. Burghardt PR, Fulk $\sqcup$, Hand GA, Wilson MA. The effects of chronic treadmill and wheel running on behavior in rats. Brain Res 2004; 1019: 84-96.

34. Koltyn KF. Analgesia following exercise: a review. Sports Med 2000; 29: 85-98.

35. Farrel PA, Garthwaite TL, Gustafson AB. Plasma adrenocorticotropin and cortisol responses to supramaximal treadmill exercise in trained an untrained males. Acta Physiol Scand 1987; 130:619-25.

36. Cannon JG, Meydani S, Fielding RA. Acute phase response in exercise. Part II: association between vitamin E, cytokines and musc i, rielding RA. Acute phase response in exercise.

77. Rosa LFPBC Vaisberg MW. Influências do exercício na resposta imune Rev Bras Med Esporte 2002: 8: 167-72

38. Gallin, Jl, Goldstein MI, Snyderman R. Inflammation: basic principles and clinical correlates. New York: Raven Gallin, Jl, Goldstein

Press, 1992; 1-4.

39. Garcia-Leme J. Hormones and inflammation. Florida: CRC Press, 1989; 165-227.

40. Martínez M, Bolaños R. Antiinflamatorios esteroideos y no esteroideos. In: Bazerque, PM, Martinez M, Maggi ND, Ceraso OL, Bolaños R. Farmacologia de la inflamación y el dolor y el dolor. Buenos Aires: Ediciones Toray Argentina, 1979; 57-85.

41. Di Rosa, M. Biological properties of carrageenan J Pharmacy Pharmacol 1971; 24: 82-102.

42. Winter CA, Risley EA, Nuss GW. Carrageenin-induced oedema in hind paw of the rat as assay for anti-inflammatory drugs. Proc Soc Exp Biol Med 1962; 111: 544-7.

43. Vane, JR. Inhibition of prostaglandin synthesis as a mechanism of action for aspirin-like drugs. Nature 1971; 231:232-7.

44. Lees P. May SA McKellar QA Pharmacology and therapeutics of non-steroidal and inflammatory drugs in dog and cat. J Small Anim Pract 1991; 32: 183-93.

45. Ostrowski K, Rohde T, Zacho M, Asp S, Pedersen BK. Evidence that IL-6 is produced in skeletal muscle during intense long term activity. J Physiol 1998; 508: 949-53.

46. Shek PN, Shephard RJ. Physical exercise as a human model of limited inflammatory response. Can J Physiol Pharmacol 1998; 76: 589-97.

47. Bury TB, Louis R, Rademecker MF, Pirnaay F. Blood mononuclear cells mobilization and cytokines secretion during prolonged exercises. Int J Sports Med 1995; 17: 156-60

48. Ostrowski K, Rohde T, Asp S, Schjerling P, Pedersen BK. Pro and anti-inflammatory cytokine balance in strenuous exercise in humans. J Physiol 1999; 515:287-91.

49. DeRijk RH, Boelen A, Tilders FJ, Bereknbosch F. induction of plasma interleukin- 6 by circulating adrenaline in the rat. Psychoneuroendocrinology 1994; 19: 155-63.

50. Turnbull AV, Rivier CL. Regulation of the hypothalamic-pituitary-adrenal axis by cytokines: actions and mechanisms of action. Physiol Rev 1999; 79: 1-85.

51. Garcia-Leme J, Wilhelm DL. The effects of adrenalectomy and corticosterone or vascular permeability response in the skin of rat. Br J Exp Pathol 1975; 56: 402-07.

52. Terman GW, Morgan MJ, Liebskind JC. Opioid and non-opioid stress analgesia from cold water swim: importance of stress severity. Brain Res 1986; 372: 167-71 